\title{
Neuroendoscopy via an Extremely Narrow Foramen of Monro: A Case Report
}

\author{
Alhusain Nagm, ${ }^{1,2}$ Toshihiro Ogiwara, ${ }^{1}$ Tetsuya Goto, ${ }^{1}$ Akihiro Chiba, ${ }^{1}$ and Kazuhiro Hongo ${ }^{1}$
}

Herein, safe and reliable neuroendoscopic biopsy via an extremely narrow foramen of Monro (ENFM) for a nonhydrocephalic patient with hypothalamic and pineal region tumors was successfully applied. A 17-year-old boy presented with hypothalamic manifestations attributed to hypothalamic and pineal region tumors. Small ventricles were seen. Intraoperatively, to advance different diameter steerable fiberscopes via ENFM, the third ventricle was flushed to induce a moment increase in the intraventricular pressure with subsequent dilatation of FM. Postoperative course was uneventful. Histopathological studies revealed a yolk sac tumor. Adjuvant therapy was applied. Follow-up neuroimaging disclosed marvellous improvement of the condition. His symptoms gradually improved.

Keywords: endoscopic biopsy; foramen of Monro; pineal tumor; small ventricle; fiberscope

\section{Introduction}

Neuroendoscopy is widely accepted as the first choice for biopsy of pediatric intra- or periventricular tumors. ${ }^{1)}$ In many cases, histological confirmation alone is adequate and lead to subsequent chemo- or radiotherapy. ${ }^{2)}$ The combination of neuroendoscopy and neuronavigation improves the accuracy of endoscopic approach and further minimizes brain trauma ${ }^{3)}$ thus, ventricular dilatation is no longer an indispensable prerequisite. ${ }^{1)}$ However, when the foramen of Monro (FM) is narrow the procedure will be more challenging. Herein, the authors presented surgical tips to perform a minimally invasive approach to the hypothalamic region tumor via an extremely narrow FM (ENFM), and obtain an accurate histolopathological diagnosis for promising excellent adjuvant therapeutic outcome.

\section{Case Report}

\section{History and examination}

A 17-year-old boy suffered from hyperphagia with subsequent increased body weight, as he gained 40 kilograms in 8 months. The detailed neuropsychological assessment revealed no deficits, apart from scattered focal visual field defects. General physical examination disclosed morbid

${ }^{1}$ Department of Neurosurgery, Shinshu University School of Medicine, Matsumoto, Japan and ${ }^{2}$ Department of Neurosurgery, Al-Azhar University Faculty of Medicine-Nasr city, Cairo, Egypt

Received: May 25, 2016; Accepted: July 18, 2016 obesity "body mass index $(B M I)=33.95$ ". Preoperative hormonal assay showed reduction of the luteinizing hormone, follicle-stimulating hormone and testosterone levels. Repeated tumor markers' assessment disclosed gradual marked elevation of the alpha-fetoprotein (AFP) $(1999.3 \mathrm{ng} / \mathrm{ml})$ and normal human chorionic gonadotropin levels. Preoperative magnetic resonance images (MRIs) revealed hypothalamic and pineal region tumors. Additionally, enhanced nodules were noticed within the left lateral and fourth ventricles (Fig. 1A). Small ventricles (Frontal and occipital horn ratio (FOR) [19] > 0.37) was seen (Fig. 1B). Fluid-attenuated inversion recovery (FLAIR) MRI disclosed marked bilateral forniceal edema, with subsequent obvious narrowing of the FM. The right FM was $1.35 \mathrm{~mm}$, and the left FM was $2.02 \mathrm{~mm}$ in diameter. Extremely narrow FM were suspected (Fig. 1C, D). A screening MRI of the spine was negative for tumor.

\section{Operation}

Under general anesthesia, the patient was positioned supine with the head elevated and flexed to minimize cerebrospinal fluid (CSF) outflow. As an enhanced nodule was located in the left lateral ventricle, and the left FM was relatively wider than the right one (Fig. 1D), the neuroendoscopic approach was performed via left precoronal burr hole. Under neuronavigation guidance, a strait trajectory was designed (to avoid undue torque on the cortex or structures around the FM) from the skull bone, passing through the ENFM to the tumor, and the burr hole was tailored accordingly $(1 \mathrm{~cm}$ anterior to the coronal suture and over the midpupillary line). After dural incision and arachnoid coagulation, the lateral ventricle was approached, with ventricular tap needle, under navigational guidance (BrainLAB, Feldkirchen, Germany). ${ }^{4}$ Only the location of the entry point and the approach trajectory were determined with neuronavigation while the procedure itself was performed under endoscopic visual control. Once the ventricle was cannulated, CSF pressure was measured and samples were sent for further analysis. Using the same trajectory, an endoscopic transparent sheath (Neuroport ${ }^{\circledR}$ mini size; Olympus Corp., Tokyo, Japan) was introduced. First, steerable fiberscope $2.5 \mathrm{~mm}$ (MACHIDA SEISAKUSYO, MACHIDA, INC, Tokyo, Japan) was advanced towards the lateral ventricle. The ventricle was like a gorge (Fig. 2E), careful dripping irrigation was performed to ensure patency of the ingress route, using the controllable endoscopic channel which was connected to a reservoir of the warm artificial CSF solution. Endoscopic inspection of the lateral 

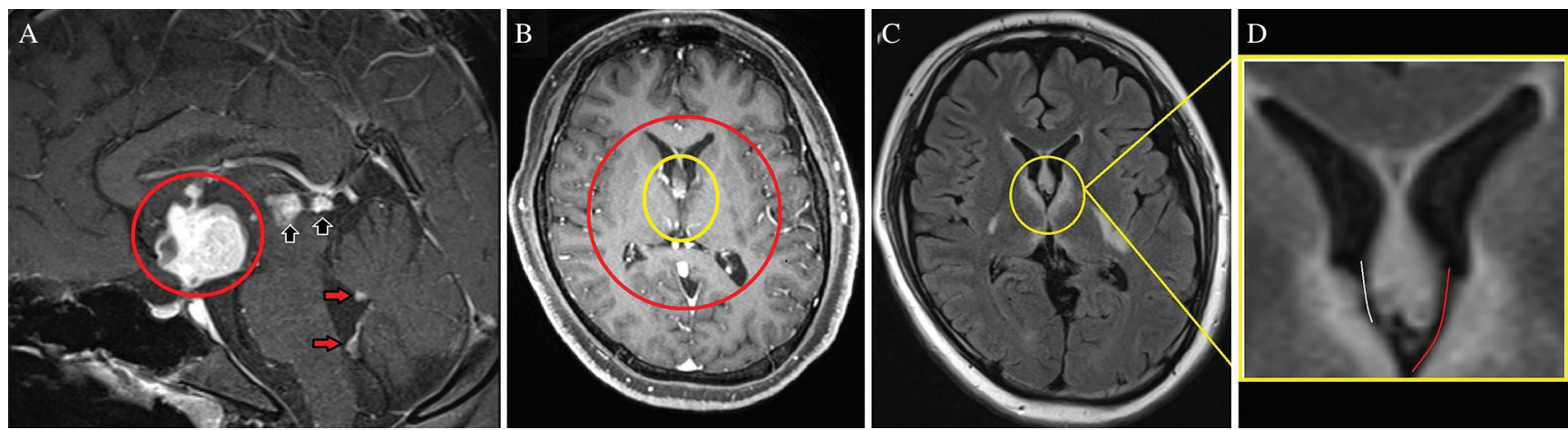

Fig. 1 Preoperative MRI; (A) Post-contrasted sagittal MRI showing the suprasellar hypothalamic tumor (red circle) measuring $29 \mathrm{~mm}$ and pineal region tumor (black arrows) measuring $9 \mathrm{~mm}$ in the maximum diameters. Fourth ventricular nodules (red arrows), indicating dissemination, are seen. (B) Post-contrasted axial MRI showing no hydrocephalus (red circle), notice the extremely narrow foramina of Monro and the slit-like third ventricle (yellow circle). (C) FLAIR-MRI axial view, showing edema of the fornix with subsequent narrowing of the foramina of Monro "left $=2.02 \mathrm{~mm}$, $r i g h t=1.35 \mathrm{~mm}$ in diameters" (yellow circle). (D) Zoom in (400\%) at the level of the extremely narrow foramina of Monro, the right route (white line) is narrower than the left one (red line).

ventricle could not identify any abnormal lesions. Major landmarks (choroid plexus passing through an ENFM to the third ventricle, and at its lower margin the point where the thalamostriate vein unites with the septal vein) were identified (Fig. 2A). Here, close to FM, the flush technique was carried-out with careful monitoring of the heart rate. In this flush technique, a direct bolus of $10 \mathrm{ml}$ warm artificial CSF solution was flushed over 4 seconds, via the endoscopic channel, to induce a moment increase in the intra-thirdventricular pressure (Fig. 3) and subsequent dilatation of the FM (Fig. 2B). This slow injection accomplished a safe gradual dilatation of the FM. ${ }^{1)}$ In synchronization with this real-time dilatation of the FM, the fiberscope was advanced to the third ventricle without traumatizing the fornix, choroid plexus or related veins (Fig. 3). Although some landmarks of the third ventricle were not distinguishable, a whitish hypothalamic tumor was clearly visualized (Fig. 2C). The $2.5 \mathrm{~mm}$ fiberscope was exchanged with $3.7 \mathrm{~mm}$ fiberscope (Fig. 2D) (Karl Storz, Tuttlingen, Germany) and an initial biopsy was taken for frozen section, followed by $5.0 \mathrm{~mm}$ videoscope (Fig. 2E-I) (Olympus Corp., Tokyo, Japan) in order to gain accurate anatomical orientation and to obtain reliable biopsies. While keeping a distance from surrounding structures, specimens were obtained from the hypothalamic lesion. As soon as the biopsy was completed, the ventricles were copiously rinsed. Local bleeding was well-controlled using irrigation. Frozen section revealed a germ cell tumor. After confirmation of hemostasis, the wound was closed without ventricular drainage. In this procedure three main surgical tips were done: 1 - neuronavigationguided ventricular tap, 2- different diameters fiberscopes for inspection and manipulation, and 3- "flush technique" to pass safely through ENFM.

\section{Postoperative course}

No evidence of surgical complications (Fig. 4A). Histopathological studies revealed a yolk sac tumor (Fig. 5). Chemotherapy (ICE: Ifosfamide-Carboplatin-Etoposide $\times 4$ cycles) followed by radiotherapy (54 Gy/30 fractions) were administered. Postoperative course was uneventful. His symptoms and the visual field gradually improved.

\section{Follow-up}

MRI 3- and 6-month-postoperatively disclosed marvelous improvement of the condition and almost disappearance of the intracranial lesions (Fig. 4B). FLAIR-MRI showed resolution of the forniceal edema and normalization of the FM (Fig. 4C). His developed panhypopituitarism, which attributed to the adjuvant therapy, is well controlled by hormonal replacements.

\section{Discussion}

\section{Neuroendoscopic biopsy}

It is most strongly indicated for tumors for which postsurgical adjuvant treatment is effective (as our case) ${ }^{5,6)}$ Because the treatment regimens for pineal region tumors vary greatly and the risks of a full resection are potentially high, an initial accurate minimally invasive tissue diagnosis is paramount. ${ }^{7}$ Biopsy may permit differentiation of cases requiring a microsurgical approach from those to be treated with adjuvant therapy alone, ${ }^{5)}$ and provide meaningful pathological data for the majority of patients. ${ }^{8-13)}$ Even, in benign or redioresistant tumors, endoscopic biopsy would lead to more appropriate management. ${ }^{2,10,14)}$

Pediatric suprasellar tumors form a unique category in histology, incidence, and treatment,${ }^{15)}$ also, present a real surgical challenge to the neurosurgeon. ${ }^{7,9,16)}$ This group of tumors do not always require aggressive surgical intervention. ${ }^{2)}$ A substantial number of neoplasms can be successfully managed without surgical intervention. ${ }^{17,18)}$ Furthermore, in cases of malignant tumors, even extensive surgical resection does not eliminate the need for subsequent adjuvant therapy. ${ }^{19,20)}$ Also, within each tumor there is the potential for heterogeneity, thus, an accurate diagnosis is pivotal in deciding on a formal treatment strategy. ${ }^{8,10,21)}$

\section{Advantages of steerable fiberscope}

Movements of only the distal portion of the endoscope allow the surgeon to avoid undue torque on the cortex or 

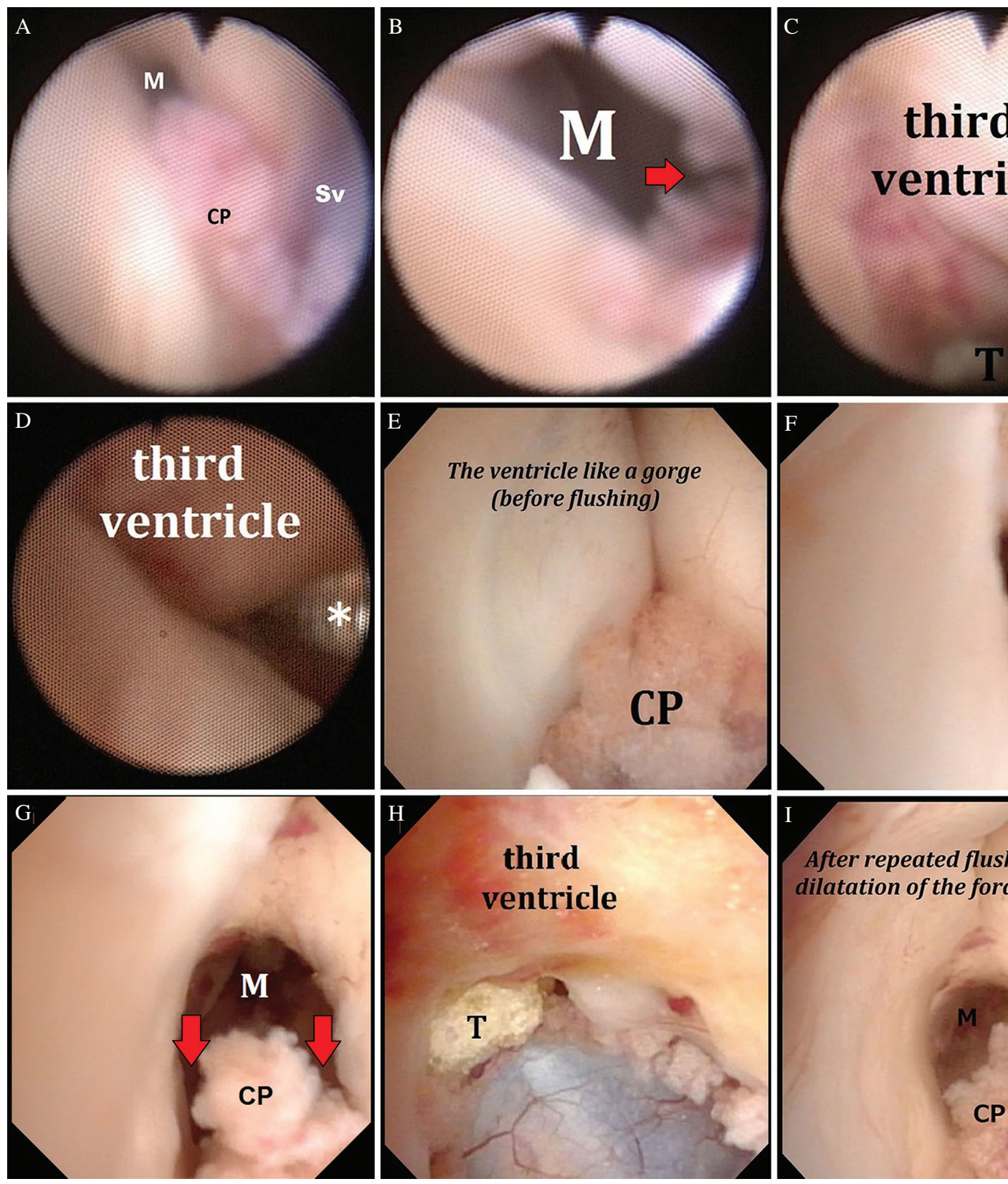

The ventricle like a gorge (before flushing)

\section{ventricle}
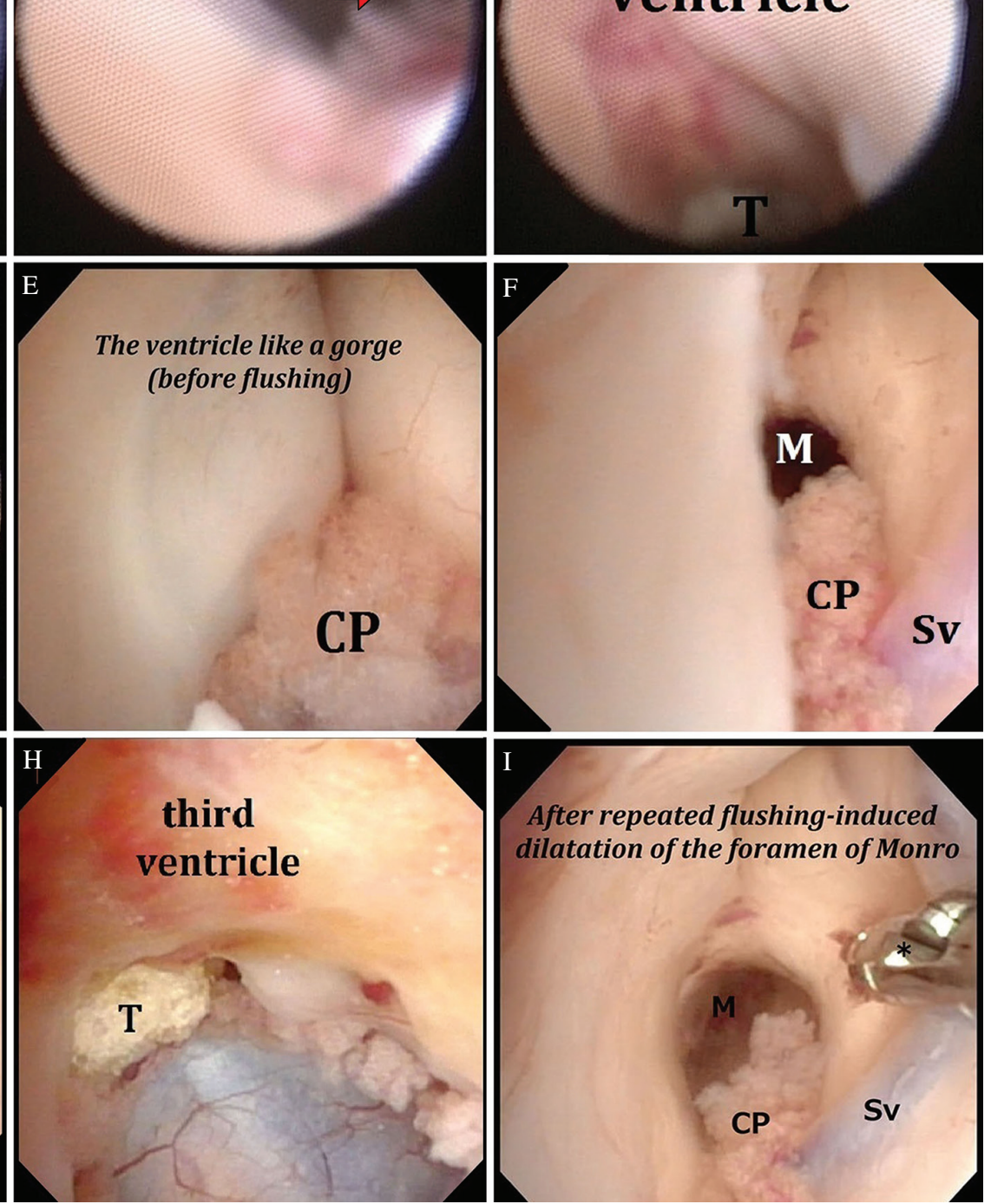

Fig. 2 Neuroendoscopic video-captured images; (A, B, C) A $2.5 \mathrm{~mm}$ fiberscope during careful endoscopic inspection. The major anatomical landmarks were identified: "the choroid plexus $(C P)$ passing through an extremely narrow $F M(M)$ to the third ventricle, and at its lower margin the point where the thalamostriate vein unites with the septal vein $(\mathrm{Sv})$ ". The whitish hypothalamic tumor (T) was visualized inside the 3rd ventricle. Notice the (image B) flushing-induced dilatation of the FM. (D) A $3.7 \mathrm{~mm}$ fiberscope was used to get tumor biopsies. (E-I) A $5.0 \mathrm{~mm}$ videoscope was introduced in order to gain accurate pertinent anatomical orientation and to obtain reliable biopsies. Before flushing the ventricle was like a gorge (E), however, during flushing the landmarks became gradually obvious (F). Just before passing through the FM to the third-ventricle: a closer position of the fiberscopes showing the efficacy of the flush technique (B and G). Notice the degree of FM dilatation and its' increased diameter ( $a$ space was created around the CP "red arrows"). Additionally, the high resolution images obtained by the 5.0 mm videoscope ruled-out any forniceal injury (I). CP: choroid plexus, M: foramen of Monro, Sv: Septal vein, T: tumor, *: tumor forceps.

structures around the FM, including the fornix, thalamus, and deep veins. ${ }^{22)}$

\section{Neuroendoscopy in small ventricles}

Small ventricles may not be served as a contraindication to endoscopic tumor biopsy. ${ }^{1,12,21-27)}$ However, without neuronavigation, the failure rate to reach the correct path to the lateral and third ventricles is high if the ventricular size is extremely small. ${ }^{15)}$

\section{Neuroendoscopic management of ENFM}

Patients without hydrocephalus are expected to all have a normal to small FM with a vulnerability to unilateral forniceal scratch, moreover, when forniceal edema is superimposed 


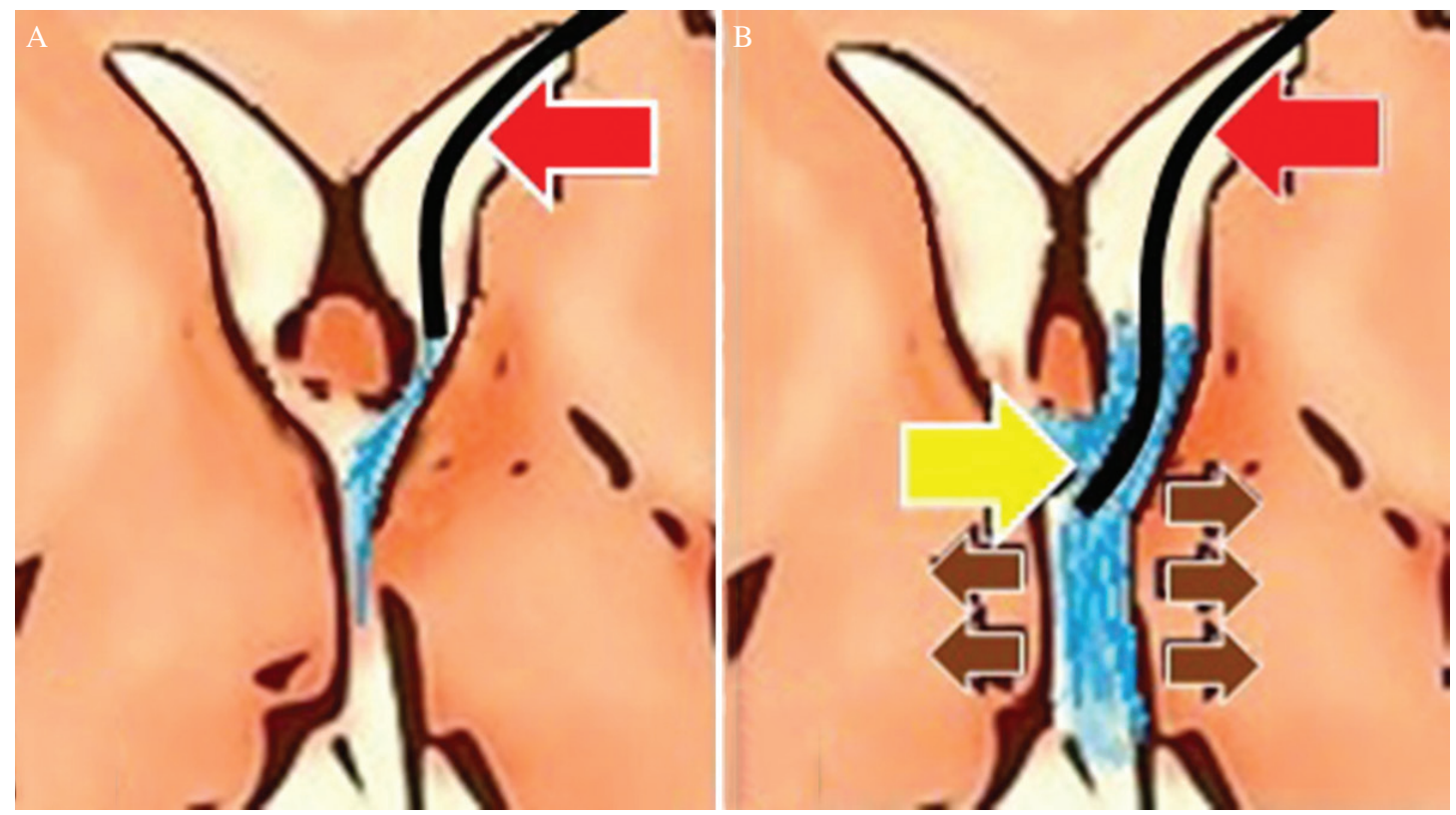

Fig. 3 Schematic drawing showing the flush technique, (A) A direct bolus of 10-ml warm artificial CSF solution was flushed gradually, via the endoscopic channel (red arrow). (B) A moment increase in the intra-third-ventricular pressure (brown arrows) and subsequent dilatation of the FM were achived. Simultaneously, the fiberscope (red arrow) was safely advanced to the third ventricle (yellow arrow). The third ventricular size became comparable.
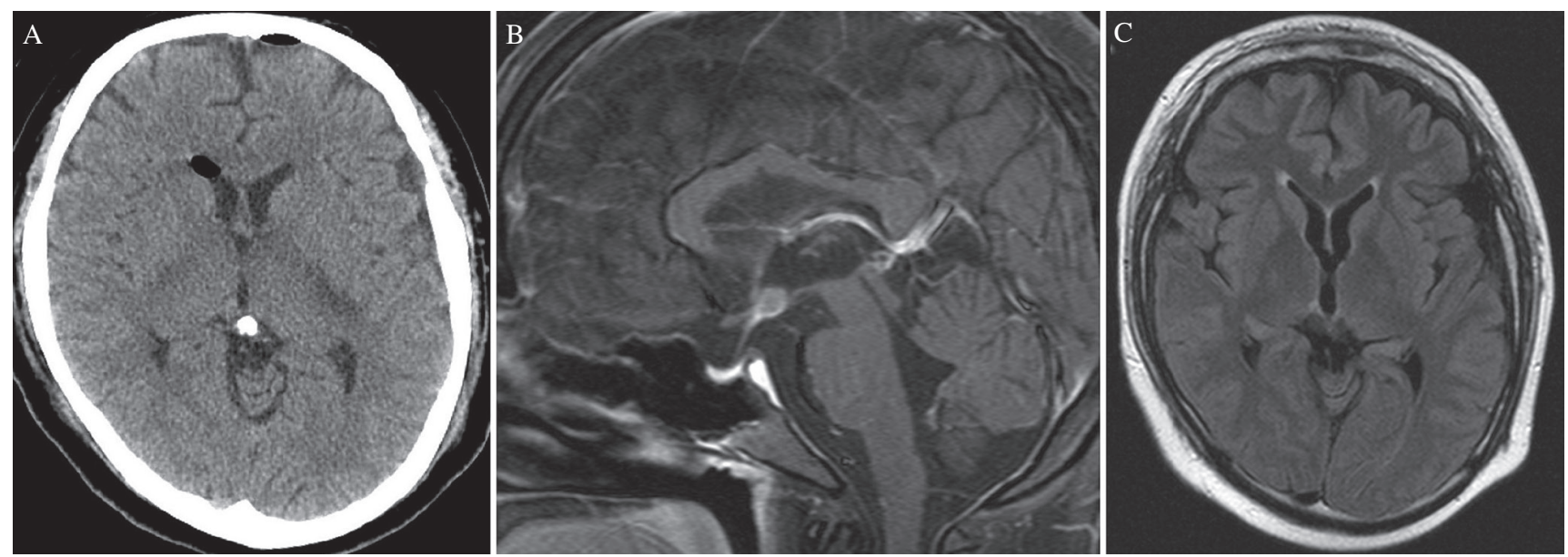

Fig. 4 Postoperative and follow-up neuroimaging; (A) CT on postoperative day 1 ruled out surgery-related complications. (B) Follow-up postcontrasted sagittal MRI 3 months postoperatively (effect of adjuvant therapy) disclosed marvelous improvement of the condition and almost disappearance of the intracranial lesions C: Follow-up FLAIR-MRI axial view 6 months postoperatively, showing resolution of the forniceal edema and normalization of the foramina of Monro (left $=4.98 \mathrm{~mm}$, right $=4.25 \mathrm{~mm}$ in diameters).

(as in our case), FM became much smaller "extremely narrow" and the fornix come to be at a critical risk for injury.

In this report, an ENFM was highly suspected, and it was considered amendable by adequate meticulous flushing during small-diameter-steerable neuroendoscopy.

In previous important neuroendoscopic studies included patients with small ventricles, ${ }^{12,26,27)}$ there was no objective definition or measurement of the ventricular size. In addition, patients were categorized into the small-ventricle and ventriculomegaly groups based on FOR in other studies. ${ }^{24,28,29)}$ In turn, up to now, the size of the FM was not clearly described.
It seems necessary to record the diameter of the FM for each patient enrolled in neuroendoscopy. This might not only serve the surgical outcome by selecting the proper endoscope or the future of neuroendoscopy by further development, but also provide a pertinent anatomical reference.

\section{Flushing technique and conventional irrigation method}

Conventional irrigation method is considered effective to insufflate small ventricles. However, during this continuous irrigation, a great attention to the patient's hemodynamic status is necessary to exclude relative bradycardia secondary 

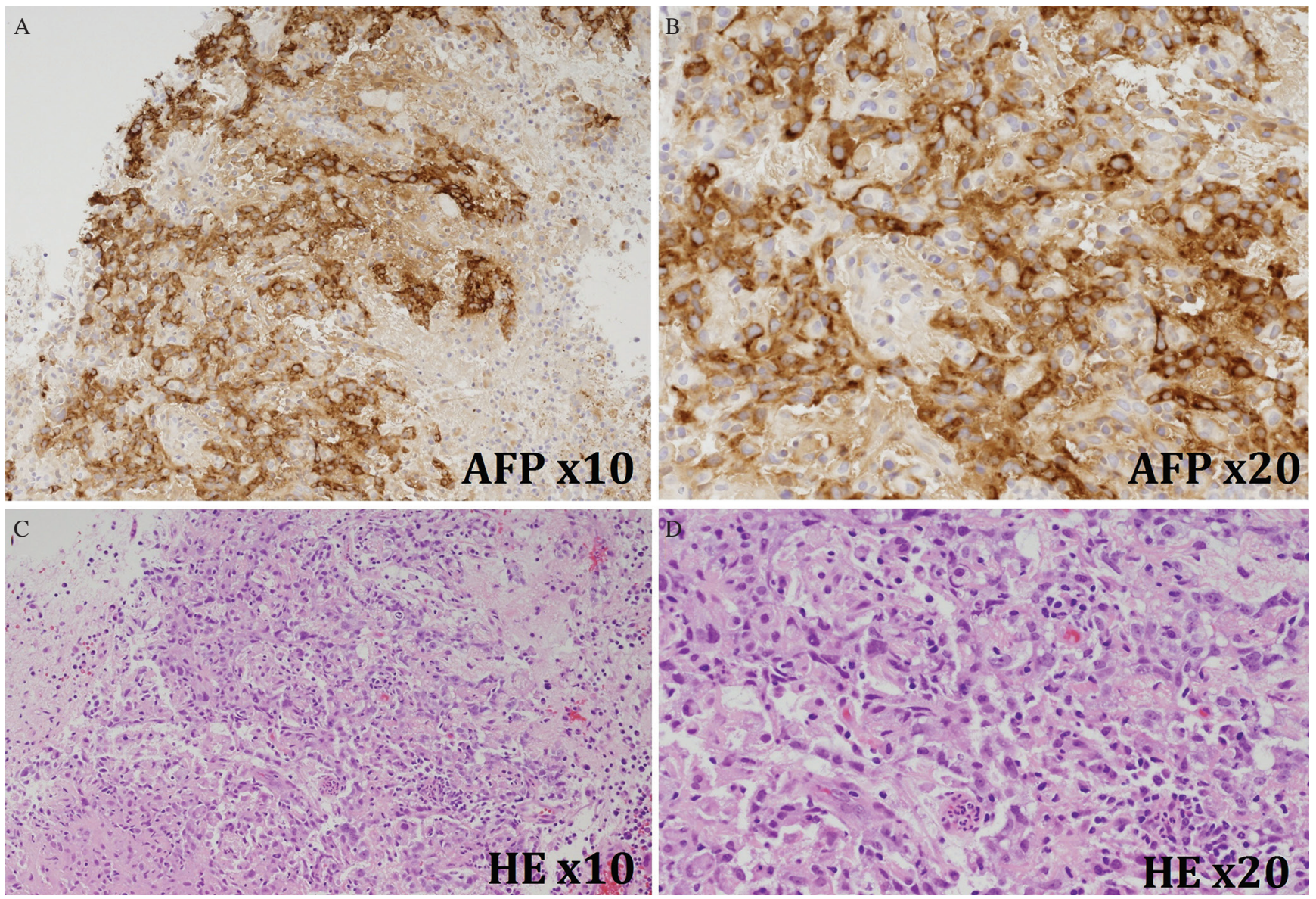

Fig. 5 Immunohistochemical and histopathological studies; (A) $(A F P \times 10)$ and $(B)(A F P \times 20)$ : Immunohistochemical staining for $\alpha$-fetoprotein (AFP), tumor cells showed strong cytoplasmic staining. (C) $(\mathrm{HE} \times 10)$ and (D) $(\mathrm{HE} \times 20)$ : Photomicrograph of histopathological sections with hematoxylin and eosin (HE) staining: small slit-like spaces lined by atypical tumor cells. Tumor cells were pleomorphic, had abundant eosinophilic or clear cytoplasm and marked nucleoli which were arranged in nest or reticular pattern. Papillary growth pattern and mitotic figures could be found.

to raised intracranial pressure (ICP). ${ }^{1)}$ In this method, the intra-lateral-ventricular pressure should be increased to ensure the ingress route while keeping the size of FM (risk for forniceal injury) unchanged. As, firstly, the exact threshold of the fluid needed to induce safe ventricular dilatation is not determined (increased ICP cannot be predicted), second, the increased ICP will be realized only once bradycardia occurs and, third, the structures around the FM still at risk, these are considered three major shortcomings in conventional method for small ventricular endoscopy.

The novel of "flush technique" exists in inducing a moment increase in the intra-third-ventricular pressure (Fig. 3 ) to amend the ENFM, before navigating the endoscope, for the safety of the structures around the FM. Thus, a nonviolent neuroendoscopic biopsy via an ENFM could be achieved without injuring the fornix. We successfully obtained this merit (Fig. 2I). Additionally, this technique maintains an ICP that cannot exceed the pressure of a fluid column equal to the length of the endoscope, provided that a constant purge is used.

The aim of this study is not to compare the conventional irrigation method versus our introduced flush technique. However, we believe that the result of our patient may provide a perspective. Finally, we are aware that a single presented case with hypothalamic lesion is a limitation for our study. Further researches incorporating patients with ENFM and other intrathird-ventricular (posteriorly "pineal" or anteriorly-located) tumors are required to validate our results and make more solid conclusions, as injury to the fornix is more likely to occur among this group.

\section{Conclusion}

We first introduce a flushing technique by which a safe neuroendoscopic biopsy can be successfully applied even via an ENFM for non-hydrocephalic patients with intraventricular and pineal region tumors. It seems necessary to record the diameter of the FM for each patient enrolled in neuroendoscopy. This pertinent anatomical reference might serve the surgical outcome by selecting the proper technique and enhance the future of neuroendoscopy.

\section{Conflicts of Interest Disclosure}

The authors have no personal, financial, or institutional interest in any of the drugs, materials, or devices in the article. All authors who are members of the Japan Neurosurgical Society (JNS) have registered online Self-reporting Conflict 
Disclosure Statement Forms through the website for JNS members.

\section{References}

1) Cappabianca P, Cinalli G, Gangemi M, Brunori A, Cavallo LM, de Divitiis E, Decq P, Delitala A, Di Rocco F, Frazee J, Godano U, Grotenhuis A, Longatti P, Mascari C, Nishihara T, Oi S, Rekate H, Schroeder HW, Souweidane MM, Spennato P, Tamburrini G, Teo C, Warf B, Zymberg ST: Application of neuroendoscopy to intraventricular lesions. Neurosurgery 62: 575-597, 2008

2) Song JH, Kong DS, Shin HJ: Feasibility of neuroendoscopic biopsy of pediatric brain tumors. Childs Nerv Syst 26: 1593-1598, 2010

3) Schroeder HW, Wagner W, Tschiltschke W, Gaab MR: Frameless neuronavigation in intracranial endoscopic neurosurgery. J Neurosurg 94: $72-79,2001$

4) Ogiwara $T$, Goto $T$, Aoyama $T$, Nagm A, Yamamoto $Y$, Hongo $K$ : Bony surface registration of navigation system in the lateral or prone position: technical note. Acta Neurochir (Wien) 157: 2017-2022, 2015

5) Hayashi N, Murai H, Ishihara S, Kitamura T, Miki T, Miwa T, Miyajima M, Nishiyama K, Ohira T, Ono S, Suzuki T, Takano S, Date I, Saeki N, Endo S: Nationwide investigation of the current status of therapeutic neuroendoscopy for ventricular and paraventriculartumors in Japan. J Neurosurg 115: 1147-1157, 2011

6) Romero FR, Bertolini EF, Seatrari A, Guerrero SS, Guerrero RB, Jr. MC: The role of neuroendoscopy in the treatment of pineal region tumors. J Bras Neurocirurg 22: 21-25, 2011

7) Ahmed AI, Zaben MJ, Mathad NV, Sparrow OC: Endoscopic biopsy and third ventriculostomy for the management of pineal region tumors. World Neurosurg 83: 543-547, 2015

8) Chernov MF, Kamikawa S, Yamane F, Ishihara S, Kub O, Hori T: Neurofiberscopic biopsy of tumors of the pineal region and posterior third ventricle: indications, technique, complications, and results. $\mathrm{Neu}$ rosurgery 59: 267-277, 2006

9) Chibbaro S, Di Rocco F, Makiese O, Reiss A, Poczos P, Mirone G, Servadei F, George B, Crafa P, Polivka M, Romano A: Neuroendoscopic management of posterior third ventricle and pineal region tumors: technique, limitation, and possible complication avoidance. Neurosurg Rev 35: 331-38, 2012

10) Constantini S, Mohanty A, Zymberg S, Cavalheiro S, Mallucci C, Hellwig D, Ersahin Y, Mori H, Mascari C, Val JA, Wagner W, Kulkarni AV, Sgouros S, Oi S: Safety and diagnostic accuracy of neuroendoscopic biopsies: an international multicenter study. J Neurosurg Pediatr 11: 704-709, 2013

11) Fukushima $T$ : Endoscopic biopsy of intraventricular tumors with the use of a ventriculofiberscope. Neurosurgery 2: 110-113, 1978

12) Gaab MR, Schroeder HW: Neuroendoscopic approach to intraventricular lesions. J Neurosurg 88: 496-505, 1998

13) Pople IK, Athanasiou TC, Sandeman DR, Coakham HB: The role of endoscopic biopsy and third ventriculostomy in the management of pineal region tumours. Br J Neurosurg 15: 305-331, 2011
14) Ellenbogen RG, Moores LE: Endoscopic management of a pineal and suprasellar germinoma with associated hydrocephalus: technical case report. Minim Invasive Neurosurg 40: 13-5; discussion 16, 1997

15) Kim K, Yeon JY, Seol HJ, Shin HJ: Transventricular endoscopic biopsy of suprasellar tumors: a pediatric case series. Childs Nerv Syst 29: 1285-91, 2013

16) Chibbaro S, Di Rocco F, Makiese O, Reiss A, Poczos P, Mirone G, Servadei F, George B, Crafa P, Polivka M, Romano A: Neuroendoscopic management of posterior third ventricle and pineal region tumors: technique, limitation, and possible complication avoidance. Neurosurg Rev 35: 331-340, 2012

17) Deopujari CE, Kumar A, Karmarkar VS, Biyani NK, Mhatre M, Shah NJ: Pediatric suprasellar lesions. J Pediatr Neurosci 6: S46-S55, 2011

18) Nicholson JC, Punt J, Hale J, Saran F, Calaminus G; Germ Cell Tumour Working Groups of the United Kingdom Children's Cancer Study Group (UKCCSG) and International Society of Paediatric Oncology (SIOP): Neurosurgical management of paediatric germ cell tumours of the central nervous system - a multi-disciplinary team approach for the new millennium. Br J Neurosurg 16: 93-95, 2002

19) Matsutani M, Sano K, Takakura K, Fujimaki T, Nakamura O, Funata N, Seto T: Primary intracranial germ cell tumors: a clinical analysis of 153 histologically verified cases. J Neurosurg 86: 446-455, 1997

20) Oi S, Kamio M, Joki T, Abe T: Neuroendoscopic anatomy and surgery in pineal region tumors: role of neuroendoscopic procedure in the 'minimally-invasive preferential' management. J Neurooncol 54: 277-286, 2001

21) Song JH, Kong DS, Seol HJ, Shin HJ: Transventricular biopsy of braintumor without hydrocephalus using neuroendoscopy with navigation. J Korean Neurosurg Soc 47: 415-419, 2010

22) Ogiwara H, Morota N: Flexible endoscopy for management of intraventricular brain tumors in patients with small ventricles. J Neurosurg Pediatr 14: 490-494, 2014

23) Lee MH, Kim HR, Seol HJ, Shin HJ: Neuroendoscopic biopsy of pediatric brain tumors with small ventricle. Childs Nerv Syst 30: 1055-1060, 2014

24) Naftel RP, Shannon CN, Reed GT, Martin R, Blount JP, Tubbs RS, Wellons JC: Small-ventricle neuroendoscopy for pediatric brain tumor management. J Neurosurg Pediatr 7: 104-110, 2011

25) Souweidane MM: Endoscopic surgery for intraventricular brain tumors in patients without hydrocephalus. Neurosurgery 57: 312-318, 2005

26) Souweidane MM, Sandberg DI, Bilsky MH, Gutin PH: Endoscopic biopsy for tumors of the third ventricle. Pediatr Neurosurg 33: 132-137, 2000

27) Yamamoto M, Oka K, Takasugi S, Hachisuka S, Miyake E, Tomonaga M: Flexible neuroendoscopy for percutaneous treatment of intraventricular lesions in the absence of hydrocephalus. Minim Invasive Neurosurg 40: 139-143, 1997

28) O'Hayon BB, Drake JM, Ossip MG, Tuli S, Clarke M: Frontal and occipital horn ratio: A linear estimate of ventricular size for multiple imaging modalities in pediatric hydrocephalus. Pediatr Neurosurg 29: 245-249, 1998

29) Souweidane MM: Endoscopic surgery for intraventricular brain tumors in patients without hydrocephalus. Neurosurgery 62: SHC1042SHC1048, 2008

Corresponding author:

Toshihiro Ogiwara, MD, Department of Neurosurgery, Shinshu University School of Medicine, 3-1-1 Asahi, Matsumoto 390-8621, Japan.

$\triangle$ togiwara@shinshu-u.ac.jp 